\title{
Metallomics
}

\section{Selected metal ions protect Bacillus subtilis biofilms from erosion $\dagger$}

Cite this: Metallomics, 2014 6,1441

Received 20th February 2014 Accepted 9th April 2014

DOI: 10.1039/c4mt00049h

www.rsc.org/metallomics

\author{
S. Grumbein, ${ }^{a b}$ M. Opitz ${ }^{c}$ and O. Lieleg ${ }^{a b}$
}

\begin{abstract}
Many problems caused by bacterial biofilms can be traced back to their high resilience towards chemical perturbations and their extraordinary sturdiness towards mechanical forces. However, the molecular mechanisms that link the mechanical properties of a biofilm with the ability of bacteria to survive in different chemical environments remain enigmatic. Here, we study the erosion stability of Bacillus subtilis (B. subtilis) biofilms in the presence of different chemical environments. We find that these biofilms can utilize the absorption of certain metal ions such as $\mathrm{Cu}^{2+}, \mathrm{Zn}^{2+}, \mathrm{Fe}^{2+}, \mathrm{Fe}^{3+}$ and $\mathrm{Al}^{3+}$ into the biofilm matrix to avoid erosion by shear forces. Interestingly, many of these metal ions are toxic for planktonic $B$. subtilis bacteria. However, their toxic activity is suppressed when the ions are absorbed into the biofilm matrix. Our experiments clearly demonstrate that the biofilm matrix has to fulfill a dual function, i.e. regulating both the mechanical properties of the biofilm and providing a selective barrier towards toxic chemicals.
\end{abstract}

\section{Introduction}

Bacteria can grow in adverse and challenging environments, e.g. in the presence of toxic substances such as disinfectants or metal ions. ${ }^{1-3}$ Yet, to do so, they need to rely on the protective abilities of self-produced biopolymers into which the bacteria embed themselves. Such a community of embedded bacteria is referred to as a bacterial biofilm. ${ }^{4-7}$ The protective abilities of those biofilm biopolymers can cause severe problems in situations where biofilms are undesirable, e.g. in health care where they can colonize catheters, implants and contact lenses and resist antibiotic treatments. ${ }^{8,9}$

Other problems due to the formation of biofilms are observed in industrial settings. There, biofilms can interrupt the cooling water supply due to plugging and corrosion of pipes, ${ }^{10}$ and biofilms on surfaces in food production enhance the risk for product contamination with pathogenic microflora. ${ }^{11}$ As a consequence, the deactivation and removal of biofilms from surfaces have become a main goal in biofilm research, and the mechanical properties of those biofilms have gained considerable attention recently. ${ }^{12-20}$

\footnotetext{
${ }^{a}$ Zentralinstitut für Medizintechnik, Technische Universität München, 85748 Garching, Germany. E-mail: oliver.lieleg@tum.de

${ }^{b}$ Fakultät für Maschinenwesen, Technische Universität München, 85748 Garching, Germany

${ }^{c}$ Fakultät für Physik, Ludwig-Maximilians-Universität München, 80333 Munich, Germany

$\dagger$ Electronic supplementary information (ESI) available. See DOI: 10.1039/ $\mathrm{c} 4 \mathrm{mt} 00049 \mathrm{~h}$
}

It has been shown that the presence of shear forces and different ionic conditions during growth can have an impact on biofilm mechanics. ${ }^{21-25}$ Also the viscoelastic properties of mature biofilms can be altered by shear forces and chemicals. ${ }^{26-29}$ When those shear forces are generated by flowing liquids, detachment of individual bacteria or erosion of biofilm fragments has been described. $^{30-34}$ The erosion stability of biofilms depends on the biofilm species ${ }^{35,36}$ and on the chemical environment the biofilm is exposed to. ${ }^{29,37-39}$ However, it is not clear whether chemical conditions that are beneficial for the mechanical properties of a biofilm might be harmful for the embedded bacteria.

Here, we analyze the erosion stability of biofilms grown by the bacterium Bacillus subtilis B1 (B. subtilis B1). B. subtilis is a soil-dwelling gram-positive bacterium, but it has recently also been found in the human gut. ${ }^{40}$ B. subtilis strains are used for biotechnology applications and have emerged as model organisms for biofilm formation. ${ }^{41,42}$ The strain used in this study was isolated from an oil field ${ }^{43}$ and can form biofilms on solid surfaces. We show that those B. subtilis B1 biofilms can protect themselves from erosion by shear forces by absorbing metal ions into the biofilm matrix. This mechanical protection is often accompanied by an increase in the shear stiffness of the biofilm that is induced by the absorbed metal ions. On top of providing this mechanical fortification, the ion absorption established by the biofilm biopolymer matrix also helps to shield the embedded bacteria from ions that are toxic for planktonic B. subtilis B1 bacteria. This illustrates that the biopolymer matrix of bacterial biofilms has to fulfill a dual function, i.e. regulating both the mechanical properties and the permeability of the biofilm. 


\section{Experimental}

\section{Biofilm formation}

B. subtilis strain $\mathrm{B} 1^{43}$ was used for all experiments. Overnight cultures were grown from a frozen glycerol stock in LB (Luria/ Miller) medium at $37{ }^{\circ} \mathrm{C}$ and $90 \mathrm{rpm}$ shaking (Certomat BS-1, Sartorius AG, Göttingen, Germany). Biofilms were grown by plating $100 \mu \mathrm{L}$ of an overnight culture onto 1.5\% (w/v) agar plates containing LB (Luria/Miller) and incubating those agar plates at $37{ }^{\circ} \mathrm{C}$ for 24 hours.

\section{Erosion assay}

Customized sample holders were crafted from PTFE plates. Each sample holder has a cylindrical hole of $20 \mathrm{~mm}$ diameter and $3 \mathrm{~mm}$ depth. Sample holders were sterilized prior to filling with $1 \mathrm{~mL}$ of $1.5 \%(\mathrm{w} / \mathrm{v})$ agar containing LB (Luria/Miller) generating a circular agar patch. Afterwards, $20 \mu \mathrm{L}$ of an overnight culture was mixed with $80 \mu \mathrm{L}$ of fresh LB and distributed over the agar surface of the sample holders. Sample holders were then incubated at $37{ }^{\circ} \mathrm{C}$ for 24 hours for biofilm growth. An example of such a biofilm-covered agar patch is depicted in Fig. 1a. To conduct the erosion tests, $25 \mathrm{~mL}$ of a testing liquid was prepared and filled into centrifuge tubes. The sample holders were placed into the centrifuge tubes (Fig. 1b) which in turn were mounted onto a lab shaker (Unimax 1010, Heidolph Instruments, Schwabach, Germany). The lab shaker was then set into rotation at $300 \mathrm{rpm}$ for defined time intervals which generated a shear stress of $\sim 1.6 \mathrm{mPa}$ (see ESI $\dagger$ ). After exposure to this shakinginduced shear force, the agar layer was carefully removed from the sample holder ensuring that the remaining biofilm layer was not disturbed. Images of the biofilm-covered agar patches were acquired using a perimeter stand at a defined height with a digital camera (Canon PowerShot SX240 HS). Images were then converted into black and white using a binarization procedure implemented in the software ImageJ, and the percentage of biofilm erosion was calculated by determining the ratio of the area covered with biofilm and the total area of the agar patch.

\section{Rheological characterization}

Rheological measurements were performed using a commercial rheometer (MCR 302, Anton Paar GmbH, Graz, Austria) with a $25 \mathrm{~mm}$ plate-plate geometry and $300 \mu \mathrm{m}$ plate separation in strain-controlled mode. Mature biofilms were removed

a
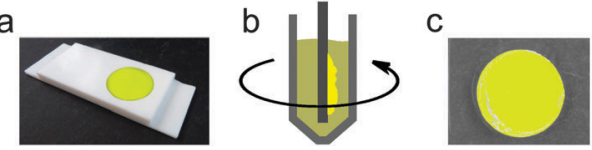

d

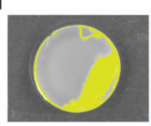

Fig. 1 Setup of the erosion experiment. A customized sample holder geometry containing a biofilm-covered agar patch (a) is inserted into a tube which is then filled with a testing solution. Shear forces are induced by setting the tube into rotational motion (b) using a lab shaker. A biofilmcovered agar patch after an erosion experiment with a very low degree of erosion is depicted in (c). A high degree of erosion correlates with a lower coverage with biofilm after exposure to shear forces as depicted in (d). For clarity, the biofilm is colored in yellow in images a-d. from agar plates by manual scraping and pooled. Small amounts of biofilm $(\sim 500 \mathrm{mg})$ were transferred to micro test tubes and weighed. $5 \%(\mathrm{v} / \mathrm{w})$ of a chemical stock solution was then added to the tubes and the chemical was distributed through the biofilm by gentle stirring with a pipette tip. Treated biofilm samples were kept at room temperature for 1 hour prior to their rheological characterization. Frequency spectra were obtained at $21{ }^{\circ} \mathrm{C}$ using small torques $(\sim 1 \mu \mathrm{Nm})$ to guarantee linear material response.

\section{Minimal inhibitory concentration}

Different ionic solutions were prepared at increasing concentrations in LB medium. We then inoculated each of those solutions with $100 \mu \mathrm{L}$ of an overnight culture of B. subtilis B1 and measured the optical densities (Victor ${ }^{3}$ Multilabel Counter, PerkinElmer, Waltham, Massachusetts, USA) of the bacterial suspensions after 24 hours of incubation at $37^{\circ} \mathrm{C}$.

\section{Regrowth assay - planktonic bacteria}

$700 \mu \mathrm{L}$ of an overnight culture of $B$. subtilis B1 was mixed with $700 \mu \mathrm{L}$ of an ionic solution in micro test tubes. Tubes were placed onto a vertical lab rotator at $2 \mathrm{rpm}$ for 1 hour to ensure good mixing. $10 \mu \mathrm{L}$ of the chemically challenged culture were then transferred into $990 \mu \mathrm{L}$ of fresh LB to remove the chemical challenge and allow for bacterial growth. Optical densities were determined (GeneQuant Pro, Amersham Biosciences, Amersham, UK) directly after mixing with fresh LB and after 15 hours of incubation at $37^{\circ} \mathrm{C}$ and $90 \mathrm{rpm}$.

\section{Regrowth assay - biofilm}

For this assay, comparable amounts of untreated biofilm $(\sim 500 \mathrm{mg}$ each) were weighed into sterile micro test tubes to ensure comparable starting conditions. Those biofilm pieces were then treated with different ionic solutions in full analogy to the chemical treatment performed for our macrorheological experiments. After an incubation time of 1 hour, the micro test tubes containing the chemically treated biofilms were rinsed on the outside with ethanol to avoid contamination and placed into $50 \mathrm{~mL}$ centrifuge tubes which then were filled with $20 \mathrm{~mL}$ of liquid LB medium. Those centrifuge tubes were then mounted onto a lab shaker and incubated at $37{ }^{\circ} \mathrm{C}$ for 15 hours to wash out bacteria from the biofilm matrix and allow for their growth. Optical densities (GeneQuant Pro) of the bacterial solutions were then obtained every hour for a total duration of 15 hours.

\section{Results}

In this study, we aim at quantifying how the erosion stability of bacterial biofilms towards shear forces depends on the chemical environment. For this purpose, we have developed a new biofilm erosion assay (Fig. 1) which employs similar principles to the macroscopic erosion test introduced by Simões et al. ${ }^{39}$ but translates them into a miniaturized version to render it more high-throughput compatible (see Experimental section for details). Fig. 1c shows a biofilm patch with a very low 
degree of erosion and Fig. 1d represents a high degree of erosion, respectively.

\section{Bacillus subtilis biofilms are sensitive to erosion by shear forces}

When the erosion test described before is performed in double distilled water $\left(\mathrm{ddH}_{2} \mathrm{O}\right)$, it takes about 5 minutes before any measurable biofilm erosion is induced. Following this initial erosion, we observe a linear increase in the biofilm removal with time. After $\sim 40$ minutes, more than $90 \%$ of the biofilm is washed off the surface, and only small chunks of biofilm material remain (Fig. 2a). A similar linear increase in the eroded biofilm mass is observed when the removed biofilm material is quantified by lyophilization, i.e. when the liquid in which the erosion assay is performed is freeze-dried and the eroded biomass is determined by weighing the lyophilized material (Fig. S1, ESI $\dagger$ ). This demonstrates that our optical analysis correctly measures the degree of biofilm removal as a function of time. Furthermore, our results show that the $B$. subtilis biofilm cannot withstand the prolonged application of shear forces in an aqueous environment. Of course, swelling of the biofilm in water could also contribute to the observed erosion process. Therefore, we repeat the same experiment with the lab shaker being switched off. In the absence of shear forces, the degree of biofilm erosion is much lower and saturates at $\sim 10 \%$ after 15 minutes (Fig. 2a). This finding verifies the suitability of our erosion assay to quantify the stability of B. subtilis B1 biofilms towards shear forces. Moreover, it demonstrates that B. subtilis $\mathrm{B} 1$ biofilms are sensitive towards shear forces as they may occur in liquid environments such as river beds or waste water pipes.

However, in their natural environment, biofilms are able to withstand shear forces, e.g. when they grow on the surface of pipes or catheters in the presence of flowing fluids. Accordingly, this indicates the existence of a mechanism that allows biofilms to protect themselves from erosion. Of course, in pipes or catheters the biofilms are exposed to a more complex chemical environment than ultrapure water. Thus, we next ask whether and how variations in the chemical composition of the aqueous environment affect the erosion stability of $B$. subtilis $\mathrm{B} 1$ biofilms.

\section{Absorption of multivalent ions protects Bacillus subtilis biofilms from erosion and increases the biofilm shear stiffness}

When growing in pipes that are typically made of copper or iron, biofilms are exposed to $\mathrm{Cu}^{2+}$ and $\mathrm{Fe}^{3+}$ ions. Thus, we next test if the presence of $\mathrm{Cu}^{2+}$ and $\mathrm{Fe}^{3+}$ ions influences the erosion stability of $B$. subtilis B1 biofilms. We repeat our shear erosion test with biofilm samples exposed to solutions of $50 \mathrm{mM} \mathrm{CuSO}_{4}$ or $50 \mathrm{mM} \mathrm{FeCl}$ and then compare the kinetics of biofilm erosion to the results obtained with ultrapure water (Fig. 2a). We find that, in the presence of $\mathrm{Cu}^{2+}$ ions, the measured biofilm erosion is comparable to when the biofilm is placed in ultrapure water without applying shear forces: over a time span of 42 minutes, less than $20 \%$ of the biofilm is removed. The virtually same stabilization effect is obtained when $\mathrm{FeCl}_{3}$ solutions are used. Apparently, both ions can stabilize the biofilm and protect it from surface erosion. In addition to this stabilization effect, we also observe a change in the material quality of the biofilm. For both ions, the color of the biofilm is altered after incubation (Fig. 2b-d): whereas the biofilm acquires an orange/brown color after exposure to $\mathrm{FeCl}_{3}$, it turns blue after exposure to $\mathrm{CuSO}_{4}$. This clearly indicates an uptake of the respective ions into the biofilm matrix. Moreover, when trying to remove the biofilm from the agar surface by manual $a$

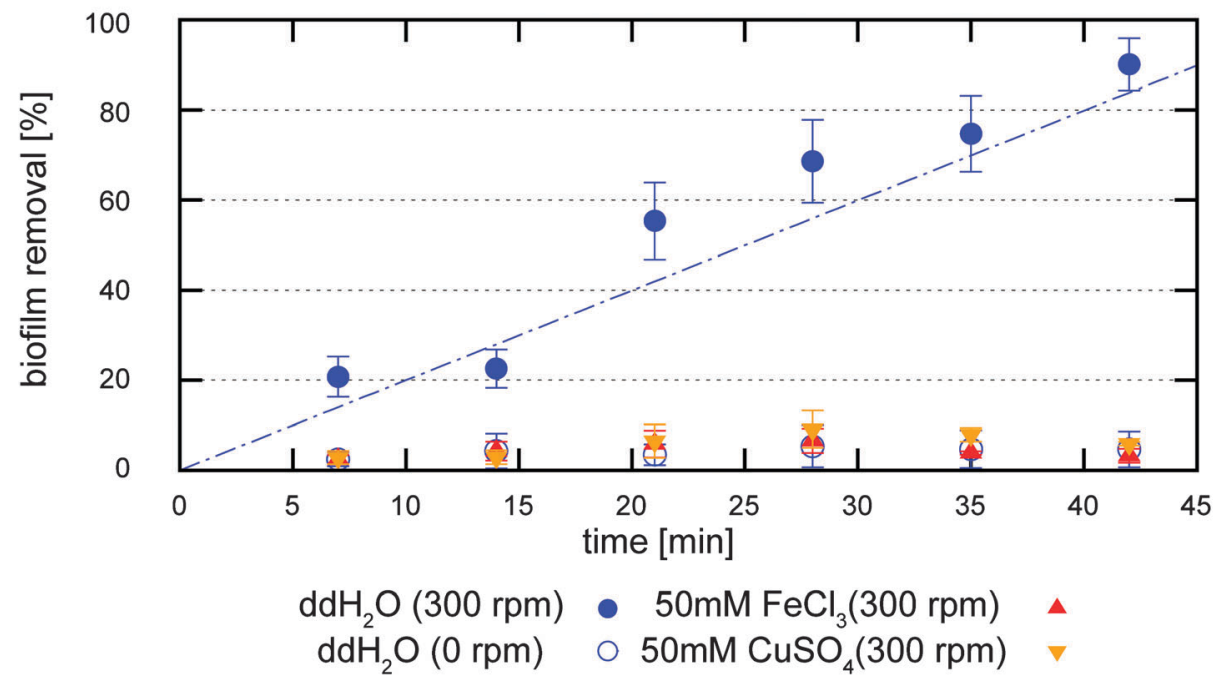

b

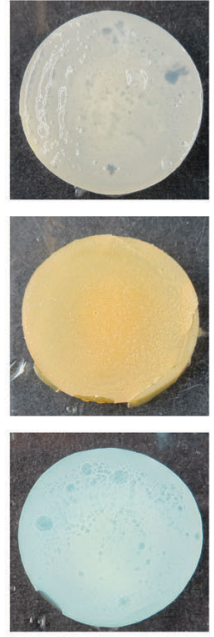

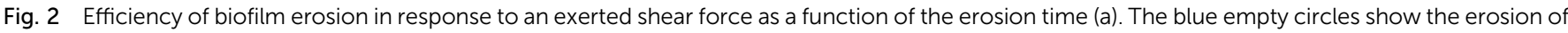

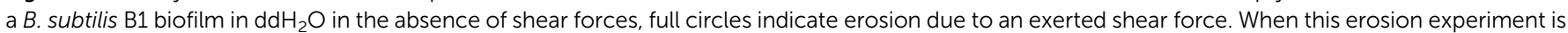

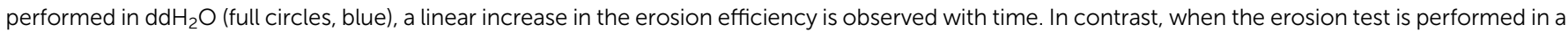

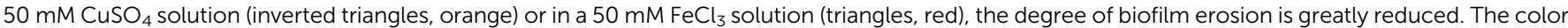

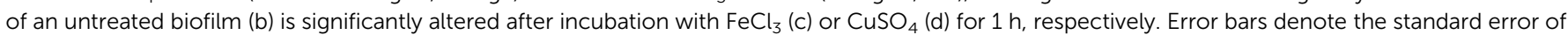
the mean as obtained from three individual samples. 
scraping, we note a significant stiffening of the biofilm matrix. This suggests that the absorption of $\mathrm{Cu}^{2+}$ and $\mathrm{Fe}^{3+}$ ions might alter the viscoelastic properties of the biofilm.

In order to quantify the putative alterations in biofilm stiffness observed from manual scraping, we next aim at measuring the viscoelastic properties of biofilms which have been exposed to selected chemicals. As a material parameter describing the mechanical properties of the biofilm in response to shear forces, we choose the dynamic shear modulus $G^{*}(f)$, a quantity that depends on the frequency of the applied shear deformation. This dynamic shear modulus is a complex quantity. Its real part, the so called storage modulus $G^{\prime}(f)$, describes the elastic properties of the biofilm whereas the imaginary part, the loss modulus $G^{\prime \prime}(f)$, represents its viscous properties. Experimentally, those two parts of the complex shear modulus are determined from the in-phase and out-ofphase part of the biofilm deformation in response to an oscillatory shear force (Fig. 3a). We measure those two parameters over a range of biologically relevant frequencies, i.e. between $10 \mathrm{mHz}$ and $10 \mathrm{~Hz}$ corresponding to time scales of $0.1 \mathrm{~s}$ to $100 \mathrm{~s}$. We observe that the storage modulus dominates over the loss modulus over the whole frequency regime tested (Fig. 3b) underscoring the pronounced elastic properties of a bacterial biofilm in contrast to the purely viscous properties of a bacterial suspension. Furthermore, we find that B. subtilis biofilms obtained from different growth batches show relatively little variation in their viscoelastic properties as we measure $G^{\prime}$ values in the range of 50 to $125 \mathrm{~Pa}$.

In order to determine the effect a given chemical might have on the viscoelastic properties of the biofilm, we prepare stock solutions and then add $5 \%(\mathrm{v} / \mathrm{w})$ of those solutions to harvested biofilms. Those chemicals are then distributed throughout the biofilm matrix by gentle stirring with a pipette tip to homogenize the sample (see Experimental section for details). As a negative control, dd $\mathrm{H}_{2} \mathrm{O}$ is added to the biofilm. By this slight dilution of the biofilm material, the storage and loss modulus are only minimally changed (Fig. 3b), and the overall shape of the frequency spectrum is unaffected. When adding $\mathrm{CuSO}_{4}$ to a harvested $B$. subtilis biofilm, the qualitative stiffening effect observed by manual scraping is confirmed: after treatment with $5 \%(\mathrm{v} / \mathrm{w})$ of $1 \mathrm{M} \mathrm{CuSO}_{4}$ (corresponding to a final $\mathrm{CuSO}_{4}$ concentration of $50 \mathrm{mM}$ as used in the erosion assay) the storage and the loss modulus are increased approximately 100 fold compared to the addition of $\mathrm{ddH}_{2} \mathrm{O}$. In comparison, when $5 \%(\mathrm{v} / \mathrm{w})$ of $1 \mathrm{M} \mathrm{FeCl}_{3}$ is added to the biofilm, the alteration in the viscoelastic moduli is even more pronounced and the resulting viscoelastic moduli are $\sim 1000$ times larger than for the treatment with $\mathrm{ddH}_{2} \mathrm{O}$ (Fig. 3b). When we calculate the loss factor of the samples, which is the ratio of the loss modulus to the storage modulus, we find no significant changes (Fig. S2, ESI $\dagger$ ).

As a next step, we test if this increase in the viscoelastic moduli occurs immediately when the biofilm is brought into contact with the metal ions, or if a certain time span is required until the maximum effect is reached. Therefore, we cover fully grown biofilms with a $50 \mathrm{mM}$ solution of $\mathrm{FeCl}_{3}$ or $\mathrm{CuSO}_{4}$ for short time intervals. We then discard the solution, harvest the exposed biofilms by scraping them from the agar surface and measure the viscoelastic properties. We observe a fast increase of both viscoelastic moduli within the first 10-15 minutes (see Fig. S4, ESI $\dagger$ ), but this increase in the biofilm viscoelasticity saturates after approximately $15 \mathrm{~min}$. However, in all those cases the dependence of the viscoelastic moduli on the shear frequency is weak. Thus, we can subsume the viscoelastic behavior of the biofilms into a single material parameter. For simplicity, $G^{\prime}(1 \mathrm{~Hz})$ is chosen as such a material parameter to compare the elastic properties of different biofilms for the remainder of this article.
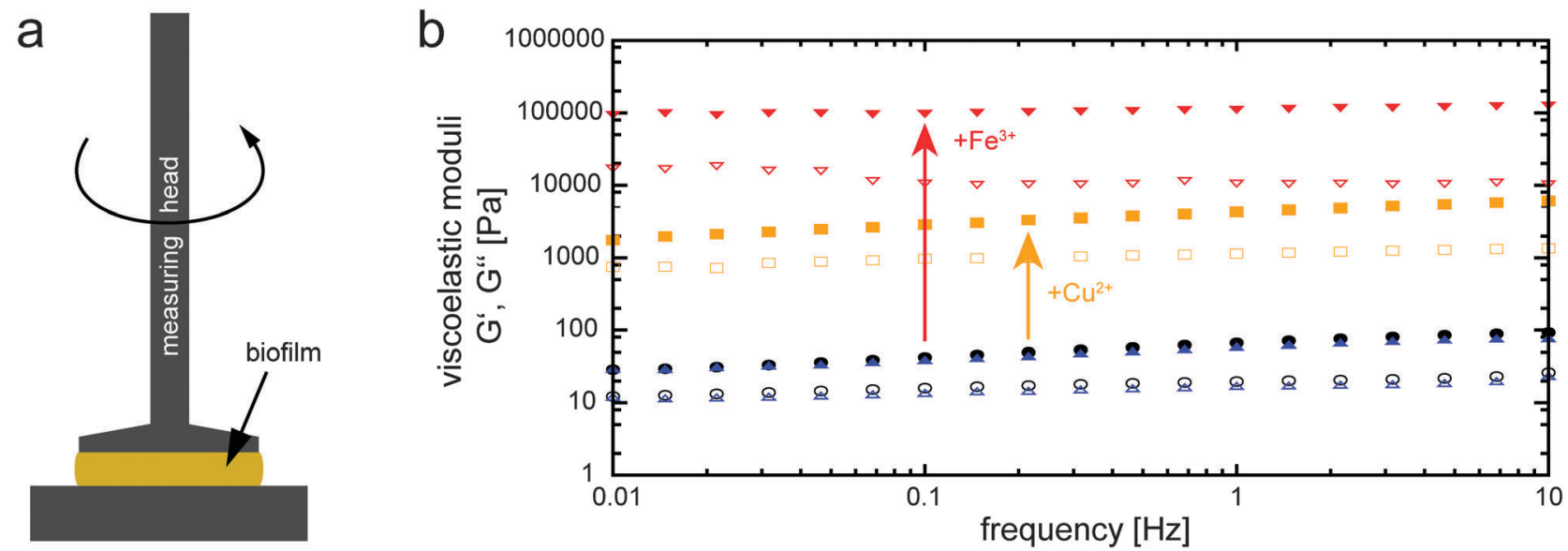

Fig. 3 Viscoelastic properties of B. subtilis B1 biofilms. (a) Measuring setup for determining the shear modulus of biofilms. An oscillatory shear force is applied and the viscoelastic properties of the biofilm are determined from the deformation response of the biofilm. (b) The viscoelastic properties of $B$. subtilis $\mathrm{B} 1$ biofilms are determined over a broad range of frequencies. We compare the viscoelastic response before (circles, black) and after treatment of biofilms with $\mathrm{dd} \mathrm{H}_{2} \mathrm{O}$ (triangles, blue), $50 \mathrm{mM} \mathrm{CuSO}_{4}$ (squares, orange) and $50 \mathrm{mM} \mathrm{FeCl}_{3}$ (inverted triangles, red). The storage modulus $\mathrm{G}^{\prime}(f)$ describing the elastic properties of the biofilm is depicted by closed symbols and the loss modulus $G^{\prime \prime}(f)$ describing the viscous properties of the biofilm is depicted by open symbols. 
a

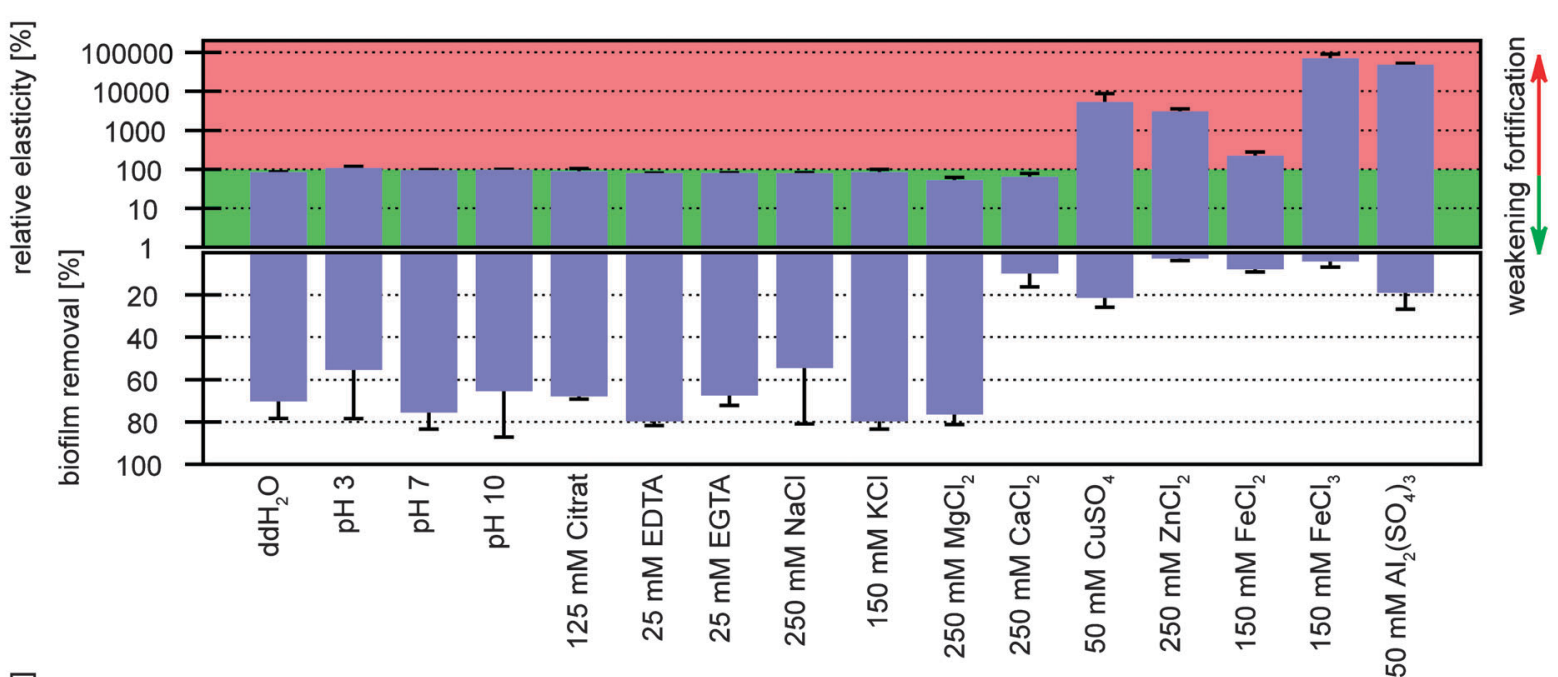

b 을
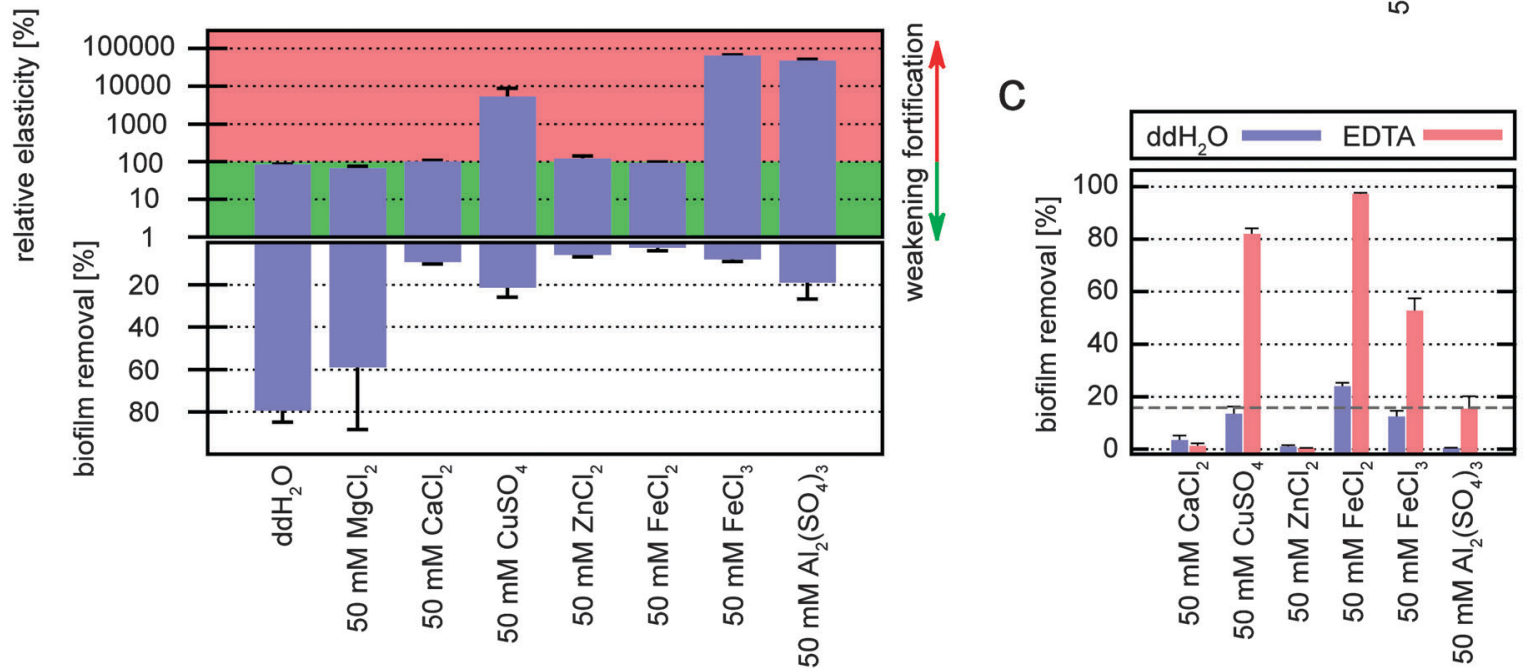

Fig. 4 Comparison of viscoelastic properties and erosion efficiency for B. subtilis biofilms. (a) Maximal influence of different chemical conditions and (b) direct comparison of identical concentrations of multivalent metal ions on the shear elasticity and erosion stability of B. subtilis B1 biofilms (see Experimental section). For all conditions in (a), stock solutions close to the solubility limit of the respective chemical were used when adding $5 \%$ (v/w) of a chemical to the biofilm matrix for rheological evaluation. The elasticity of the biofilm (upper panel) has been normalized by the elastic modulus obtained for an untreated biofilm sample. Values in the red area depict a mechanical fortification of the biofilm whereas values in the green area depict a weakening of the biofilm elasticity compared to an untreated biofilm sample. Using identical chemical conditions to that for the rheological screening, the erosion assay is performed on B. subtilis B1 biofilms. The ensuing erosion stability of biofilms is quantified by determining differences in the biofilm removal efficiencies after an erosion time of 42 minutes (lower panel). The same experimental conditions apply to (b). (c) Comparison of the erosion stability of pre-treated $B$. subtilis biofilms in distilled water ( $\mathrm{ddH}_{2} \mathrm{O}$, blue bars) and in a $25 \mathrm{mM}$ EDTA solution (red bars). The biofilm samples have been incubated in a $50 \mathrm{mM}$ solution of metal ions prior to the determination of the erosion stability. The dashed line indicates the erosion of an untreated sample in $\mathrm{ddH}_{2} \mathrm{O}$. Error bars denote the standard error of the mean as obtained from three individual samples.

Both $\mathrm{CuSO}_{4}$ and $\mathrm{FeCl}_{3}$ form acidic solutions in water. Thus, it is a priori not clear whether the observed alterations of the viscoelastic biofilm properties are due to the ions in the aqueous solution or whether they are caused by $\mathrm{pH}$ differences.

To clarify this, we repeat our rheological experiment with biofilms that have been treated with $5 \%(\mathrm{v} / \mathrm{w})$ of aqueous solutions buffered to $\mathrm{pH} 3,7$ or 10 .

We find that the viscoelastic properties of the biofilm remain unaffected by those $\mathrm{pH}$ alterations (Fig. 4a, upper panel and Table S2, ESI $\dagger$ ). This demonstrates that the changes in biofilm stiffness observed above are indeed caused by the ions in the $\mathrm{CuSO}_{4}$ and $\mathrm{FeCl}_{3}$ solution.
Can such a stiffening of the biofilm matrix only be induced by $\mathrm{Cu}^{2+}$ and $\mathrm{Fe}^{3+}$ ions or have other ions a similar effect? To answer this question, we next perform a rheological screening testing the viscoelastic properties of biofilms treated with other metal ions ranging from monovalent ions such as $\mathrm{Na}^{+}$and $\mathrm{K}^{+}$to multivalent ions such as $\mathrm{Zn}^{2+}$ or $\mathrm{Al}^{3+}$. As the naturally grown biofilm might already contain a significant amount of ions, we also test chelating agents such as citrate, EDTA and EGTA. For all those conditions we try to maximize putative effects by using stock solutions close to the solubility limit of the respective chemical when adding $5 \%(\mathrm{v} / \mathrm{w})$ of a chemical to the biofilm matrix for rheological evaluation. However, for most chemicals 
we do not detect a relevant change in the biofilm elasticity (Fig. 4a, upper panel and Table S2, ESI $\dagger$ ): neither chelating agents nor monovalent ions significantly alter the elastic properties of the biofilm. However, $\mathrm{Zn}^{2+}$ and $\mathrm{Al}^{3+}$ induce a strong fortification of the biofilm matrix similar to what we observe for biofilms treated with $\mathrm{Cu}^{2+}$ and $\mathrm{Fe}^{3+}$. In contrast, for the reduced form of iron ions (i.e. $\mathrm{Fe}^{2+}$ ), the biofilm elasticity is only weakly increased, and other divalent ions such as $\mathrm{Mg}^{2+}$ and $\mathrm{Ca}^{2+}$ hardly alter the biofilm elasticity at all.

The observation that both $\mathrm{Cu}^{2+}$ and $\mathrm{Fe}^{3+}$ ions can have a dual effect on the biofilm, i.e. enhancing the biofilm stiffness and preventing biofilm erosion, suggests that a change in the bulk material properties of the biofilm might be responsible for the erosion protection of biofilms formed by the strain Bacillus subtilis B1. To test this hypothesis, we repeat our erosion assay and quantify the biofilm erosion stability for the whole range of chemical conditions screened with macrorheology. To ensure comparable situations, the concentrations of the aqueous solutions for the erosion assay were chosen such that they match the final concentrations of the chemical agents in the biofilm as used in the rheological screening assay. We find that, in addition to $\mathrm{Cu}^{2+}$ and $\mathrm{Fe}^{3+}$ ions, also the multivalent ions $\mathrm{Ca}^{2+}, \mathrm{Zn}^{2+}, \mathrm{Fe}^{2+}$ and $\mathrm{Al}^{3+}$ significantly reduce biofilm erosion (Fig. 4a, lower panel). In contrast, for all other ions and test conditions the biofilm erosion is comparable to that obtained in pure water.

For most conditions tested so far, we have observed that the absorption of metal ions into the biofilm matrix can both induce a stiffening of the biofilm matrix and protect the biofilm layer from erosion. This seems to suggest that these two phenomena are related. However, in the case of $\mathrm{Ca}^{2+}$ ions we have observed a protection from erosion without a measurable increase in the biofilm shear stiffness. This indicates that other biofilm properties besides their shear stiffness might play a role in their stability towards shear forces induced by fluid flow.

We further test this hypothesis by repeating our evaluation of the biofilm shear stiffness and its erosion stability using lower concentrations of the metal ions tested so far. Our rationale is that the increase in the biofilm shear stiffness might depend on the ion concentration, and that below a critical biofilm stiffness the erosion protection will fail. However, if the biofilm would still be protected from erosion at those lower ion concentrations without showing increased shear stiffness, this would underscore our notion that another, independent biofilm property needs to be considered to understand its erosion resistance. For this set of experiments, we focus on multivalent ions since only those showed significant effects in our previous experiments. We compare the influence of those metal ions at identical concentrations of $50 \mathrm{mM}$, which also allows us to quantitatively compare the efficiency of those ions. We find that - in contrast to $\mathrm{CuSO}_{4}, \mathrm{FeCl}_{3}$ and $\mathrm{Al}_{2}\left(\mathrm{SO}_{4}\right)_{3}-$ the addition of $50 \mathrm{mM} \mathrm{ZnCl}$ or $\mathrm{FeCl}_{2}$ does not induce a significant increase in the biofilm shear stiffness anymore. Yet, the degree of biofilm erosion remains very low (Fig. 4b and Table S2, ESI $\dagger$ ). In conclusion, we have now observed several conditions where erosion protection is achieved without a significant alteration in the biofilm shear stiffness. This shows that, whereas the absorption of ions into a biofilm matrix can simultaneously influence the shear stiffness of a biofilm as well as its erosion stability, those biofilm properties are not necessarily linked.

\section{Selected metal ions lose their toxicity when they are absorbed in the biofilm matrix}

So far, we have described how the absorption of selected multivalent ions into the biofilm matrix can both enhance the shear stiffness of the biofilm and protect the biofilm from erosion. However, those absorbed ions might have an adverse effect on the embedded biofilm bacteria. In general, the metabolism of many bacteria requires certain metal ions, e.g. $\mathrm{Fe}^{3+}$, at low concentrations. ${ }^{44}$ Yet, at high concentrations, $\mathrm{Fe}^{3+}$ ions may become toxic - at least for bacteria in their planktonic state, i.e. when they are freely floating in a liquid environment. One could imagine that the erosion protection of the biofilm comes at a cost: does a certain percentage of the biofilm bacteria have to sacrifice their life for the greater good? To answer this question, we next test if $\mathrm{Fe}^{3+}$ ions (or the other metal ions tested here) are toxic for $B$. subtilis B1 bacteria. In principle, there are two possibilities how a given chemical might harm bacteria. First, the chemical might inhibit bacterial growth, and second the chemical might induce bacterial death. To address the first possibility, we determine the minimal inhibitory concentration (MIC) of the different metal ions by employing a growth assay using planktonic bacteria of B. subtilis B1 (see Experimental section for details). As depicted in Fig. 5, bacterial growth is not impaired by $\mathrm{NaCl}, \mathrm{CaCl}_{2}$ and $\mathrm{MgCl}_{2}$ up to concentrations of $\sim 200 \mathrm{mM}$. In contrast, we observe a strong reduction of bacterial growth for $\mathrm{FeCl}_{2}, \mathrm{FeCl}_{3}, \mathrm{CuSO}_{4}, \mathrm{ZnCl}_{2}$ and $\mathrm{Al}_{2}\left(\mathrm{SO}_{4}\right)_{3}$ even at concentrations of $10 \mathrm{mM}$ or less. This indicates that the concentrations of certain metal ions used in our previous assays, i.e. in the erosion test and macrorheological screening is already high enough to inhibit growth of planktonic bacteria; in other words, they are bacteriostatic.

Besides their bacteriostatic activity, some of the metal ions studied here might even be lethal for the B. subtilis B1 bacteria. To test this, we set up a regrowth assay in which we first perform a chemical challenge by incubating planktonic bacteria in different ionic solutions and then measure the ability of the bacteria to grow again when the chemical stress is released by transferring the bacteria into standard LB medium (see Experimental section for details). Then, the optical densities of the bacterial solutions are obtained after 15 hours of incubation in LB medium at $37^{\circ} \mathrm{C}$. Our results show that for $\mathrm{NaCl}, \mathrm{CaCl}_{2}$ and $\mathrm{MgCl}_{2}$ the bacteria are able to grow again when the chemical challenge is released. The same result is obtained for a transient chemical challenge with $\mathrm{FeCl}_{2}$ : even though $\mathrm{FeCl}_{2}$ can inhibit the growth of B. subtilis B1, once the chemical pressure is released, the optical density of the bacteria solution increases identically to that of planktonic bacteria which have not been chemically challenged (Table 1 and Table S1, ESI $\dagger$ ). In contrast, when the chemical challenge is performed with $\mathrm{FeCl}_{3}, \mathrm{CuSO}_{4}, \mathrm{ZnCl}_{2}$ or $\mathrm{Al}_{2}\left(\mathrm{SO}_{4}\right)_{3}$, we observe a significantly weaker bacterial growth when the bacteria are transferred into LB medium. This indicates that this group of 


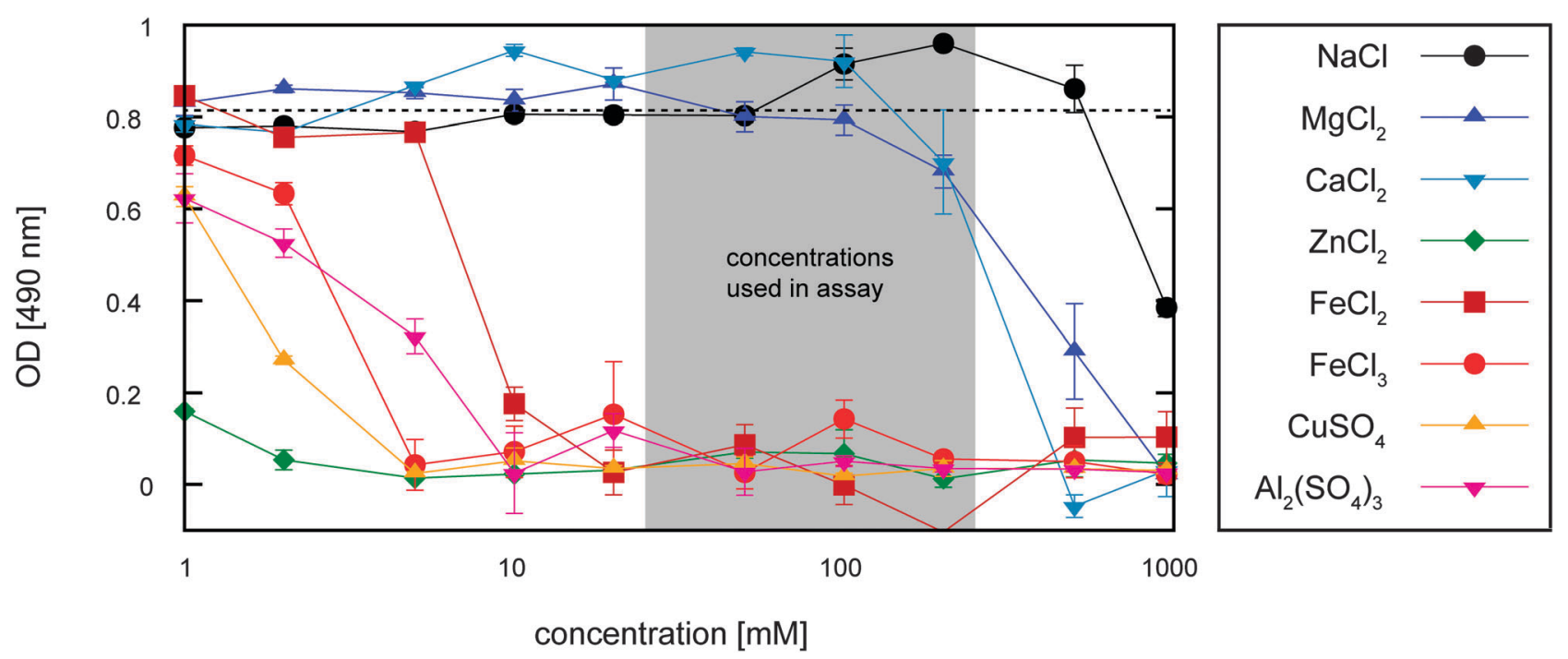

Fig. 5 Minimal Inhibitory Concentration (MIC) assay with planktonic B. subtilis B1 bacteria. LB Medium containing increasing concentrations of different metal ions was inoculated with planktonic $B$. subtilis bacteria. The optical density (OD) is determined after incubating the cultures for 24 hours. The gray area indicates the concentrations of metal ions used in our macrorheological tests and erosion assays, and the dashed line indicates the OD of a culture grown in pure media without the addition of metal ions. Error bars denote the standard error of the mean as obtained from six individual samples.

Table 1 Viability of planktonic and biofilm embedded cells

\begin{tabular}{|c|c|c|c|c|c|c|c|c|c|}
\hline & $\mathrm{dd}_{2} \mathrm{O}$ & $\begin{array}{l}250 \mathrm{mM} \\
\mathrm{NaCl}\end{array}$ & $\begin{array}{l}250 \mathrm{mM} \\
\mathrm{CaCl}_{2}\end{array}$ & $\begin{array}{l}250 \mathrm{mM} \\
\mathrm{MgCl}_{2}\end{array}$ & $\begin{array}{l}150 \mathrm{mM} \\
\mathrm{FeCl}_{2}\end{array}$ & $\begin{array}{l}150 \mathrm{mM} \\
\mathrm{FeCl}_{3}\end{array}$ & $\begin{array}{l}50 \mathrm{mM} \\
\mathrm{CuSO}_{4}\end{array}$ & $\begin{array}{l}50 \mathrm{mM} \\
\mathrm{Al}_{2}\left(\mathrm{SO}_{4}\right)_{3}\end{array}$ & $\begin{array}{l}50 \mathrm{mM} \\
\mathrm{ZnCl}_{2}\end{array}$ \\
\hline Planktonic B. subtilis $\mathrm{B} 1$ & + & + & + & + & + & - & - & - & - \\
\hline
\end{tabular}

metal ions is not only bacteriostatic but can even induce death of planktonic B. subtilis B1 bacteria - at least at high enough concentrations.

However, in a biofilm bacteria are embedded in a biopolymer matrix which might shield the microorganisms from toxic agents. Such a shielding effect has already been observed for several bacterial species where the tolerance of a mature biofilm towards antibiotics is much higher than for planktonic bacteria. We hypothesize that a similar protective mechanism towards toxic metal ions might also occur for B. subtilis B1 bacteria that reside inside a biofilm matrix.

To test this hypothesis, we repeat our regrowth assay using defined amounts of a chemically pre-treated biofilm instead of chemically challenged planktonic bacteria. Similar to our previous assay, we then measure the ability of bacteria to grow when the chemical stress is released (see Experimental section for details). Indeed, when the regrowth kinetics of different chemically challenged biofilm samples are compared to that of an untreated biofilm, we find a very similar increase in the optical density for almost all chemical challenges tested (Table 1 and Fig. S3, ESI $\dagger$ ). Only when a B. subtilis B1 biofilm is pre-treated with $250 \mathrm{mM} \mathrm{ZnCl}_{2}$, the increase in the optical density over time is weak and saturates at a final value that is $\sim 5$ times lower than the one obtained for all other conditions. Apparently, there are chemicals that can permanently suppress bacterial growth in a biofilm after a transient chemical challenge suggesting that a large fraction of the biofilm bacteria have been killed. Most metal ions, however, seem to be trapped in the biofilm matrix in such a way that they are not able to harm the bacteria and can therefore increase the resistance of the biofilm towards shear forces without decimating the bacterial population.

The erosion protection of absorbed metal ions can be partially countered by the chelating agent EDTA

So far we have shown that the biofilm matrix can absorb metal ions, prevent putative toxic ions from harming the embedded biofilm bacteria and even protect the biofilm from mechanical erosion. However, in a biological or medical setting, the chemical composition of the surrounding liquid might significantly vary over time. Thus, we next ask whether the erosion protection observed so far can persist once the biofilm is exposed to a different chemical environment where the stabilizing metal ions are not present anymore. For this experiment we pre-treat the biofilm with selected solutions of metal ions prior to performing the erosion assay. This pre-treatment is performed by incubating the biofilm-covered agar patches in a solution containing $50 \mathrm{mM}$ of a metal ion for $\sim 40$ minutes. After this incubation time, the samples are transferred into $\mathrm{dd}_{2} \mathrm{O}$ and the erosion assay is performed as in our previous experiments. As depicted in Fig. 4c, the stabilizing effect induced by the metal ions indeed persists when the biofilm sample is transferred into $\mathrm{ddH}_{2} \mathrm{O}$. This indicates that the absorbed metal ions are strongly bound by 
the biofilm matrix and that they cannot easily be washed out again by pure water. However, a strong chelating agent might still be able to remove the ions from the biofilm matrix. If this indeed is possible, then the initial properties of the biofilm should be restored and the biofilm should become erosionsensitive again. To test this hypothesis, we transfer the pretreated biofilm samples into a $25 \mathrm{mM}$ EDTA solution instead of distilled water and test the erosion stability of those pre-treated biofilms in the presence of this chelating agent. EDTA is known to form highly stable chelate complexes with metal ions and thus might be able to remove the metal ions from the biofilm matrix. We find that for biofilm samples that have been pretreated with $\mathrm{Al}^{3+}, \mathrm{Ca}^{2+}$ or $\mathrm{Zn}^{2+}$ ions, the stabilizing effect remains even when the erosion assay is performed in the EDTA solution (Fig. 4c). In contrast, when $\mathrm{Fe}^{2+}, \mathrm{Fe}^{3+}$ or $\mathrm{Cu}^{2+}$ ions are used for biofilm pre-treatment, the biofilm color is reverted to that of an untreated biofilm after incubation with the EDTA solution. In addition to this change of the biofilm color, the biofilms also become erosion sensitive again and more than $50 \%$ of the biofilm can be removed in our erosion assay.

\section{Discussion}

In this study, we have developed a new assay to measure the stability of bacterial biofilms towards erosion by shear forces. With this assay, we are able to quantify the time-dependent biofilm erosion process in the presence of different metal ions. As a model system for bacterial biofilms, we have studied biofilms formed by the non-pathogenic strain B. subtilis B1. A great advantage of the B. subtilis B1 strain used here is the large amount of biomass the strain produces within a short time. With a sufficient supply of nutrition, about $1500 \mathrm{mg}$ of biofilm material can be harvested from a standard petri dish after 24 hours of incubation which is very convenient for a macrorheological screening assay as performed here. Of course, our assay could also be applied to biofilms that are generated by other bacteria provided that they grow reasonably well on agar. Such other biofilm types may include those formed by pathogenic bacteria including Pseudomonas aeruginosa or Vibrio cholerae.

The assay presented here has several advantages compared to other methods that have been introduced to study biofilm erosion: microfluidic erosion tests in microcapillaries ${ }^{45}$ may allow for following the dynamic detachment process of bacterial microcolonies from the biofilm surface, but cannot that easily be used to quantitatively compare the influence of different chemical environments on the erosion process. Such a quantitative comparison is possible with the macroscopic erosion assay introduced by Simões et al. ${ }^{39}$ which measures the decrease in biomass of biofilms that are grown on metal cylinders with a surface of $34.6 \mathrm{~cm}^{2}$. Those cylinders are rotated in a chemical bath to induce shear forces on the biofilm surface. However, this macro-assay is very time consuming as it requires several days to grow a biofilm layer of sufficient thickness on the metal cylinders. Thus, it is not a convenient platform for screening a broad range of chemical conditions. In our miniaturized erosion assay, we optically determine the decrease of biofilm coverage on test geometries with a total biofilm area of $3.1 \mathrm{~cm}^{2}$. The processing of the digital pictures obtained after shear force application is fast and robust and should also be suitable for automization. Therefore, our erosion assay constitutes a costefficient and easy-to-handle platform for high-throughput screening of chemicals that are considered for biofilm removal strategies.

Using this erosion assay in combination with macrorheological measurements, we have shown that the absorption of metal ions into the biofilm matrix can both induce a stiffening of the biofilm matrix and protect the biofilm layer from erosion. We have observed significant erosion protection for ion concentrations of $50 \mathrm{mM}$ and higher. Although the ion concentrations used here are larger than typical release values of metal ions in water distribution pipes, ${ }^{46}$ an accumulation of those ions in the biofilm matrix beyond the ion concentrations found in the aqueous medium might still occur. Indeed, we find a first indication for such an oversaturation

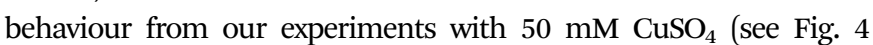
and Fig. S4, ESI $\dagger$ ): when the biofilm is covered with the $50 \mathrm{mM}$ $\mathrm{CuSO}_{4}$ solution for more than 20 minutes, a 10 fold higher biofilm shear stiffness is achieved compared to when $50 \mathrm{mM}$ of $\mathrm{CuSO}_{4}$ is directly forced into the biofilm by mechanical stirring. This suggests that the concentration of absorbed $\mathrm{Cu}^{2+}$ ions in the biofilm is larger than the concentration of $\mathrm{Cu}^{2+}$ ions in the surrounding liquid. Of course, this scenario would require the metal ions to have a high affinity for the biofilm matrix components - which agrees with our finding that the chelating agent EDTA cannot easily wash out all absorbed metal ions (see Fig. 4c).

One possible explanation for the increased matrix stiffness could be a simple ionic cross-linking of the biofilm matrix by the metal cations. ${ }^{47}$ Indeed, the polyanionic biopolymer poly- $\gamma$ glutamate is a main component of the biofilm matrix formed by B. subtilis $\mathrm{B} 1,{ }^{48}$ so ionic cross-linking should be possible per se. Also Pseudomonas aeruginosa biofilms can be mechanically fortified by selected metal ions, ${ }^{27,49}$ and the polyanionic biopolymer alginate has been put forward as a main component of mucoid $P$. aeruginosa biofilms. However, for those biofilms ionic cross-linking was not sufficient to account for the observed increase in biofilm stiffness upon exposure to metal ions since only trivalent and not divalent ions had a significant effect. For the B. subtilis biofilms studied here, we find an even higher degree of specificity as only selected metal ions induce a mechanical fortification and erosion protection whereas other metal ions of the same valency do not.

Furthermore, a detailed comparison of different metal ions revealed that an increase in the biofilm shear stiffness is not necessarily linked to a reduction of biofilm erosion. It seems probable that the adhesion strength of the biofilm to the surface it has been grown on is a second important parameter that needs to be addressed to understand how the stability of a bacterial biofilm towards surface erosion is regulated on a molecular scale. This question and how the adhesion strength of bacterial biofilms can be tuned by different ionic conditions needs to be addressed in future investigations.

Interestingly, neither the viscoelastic properties of a biofilm ${ }^{27,50}$ nor its erosion stability ${ }^{19}$ are directly linked to the viability of the embedded bacteria. Nevertheless, in response to the exposure to toxic ion concentrations, sporulation could be induced for a fraction 
of the embedded bacteria population, and such a sporulation event might alter the mechanical properties of the biofilm. However, as most of our experiments are performed within a time-period smaller than the time needed for spore formation in $B$. subtilis,${ }^{51}$ this is not a likely scenario. In fact, biofilm-embedded bacteria are more resistant towards heavy metals than planktonic cells, ${ }^{52}$ and biofilm cells bind metals in different quantities than planktonic cells. ${ }^{53}$ By absorbing metal ions into the biofilm matrix in such a way that the embedded bacteria are not harmed, a $B$. subtilis biofilm can make use of toxic ions to optimize its mechanical properties.

This ability of $B$. subtilis B1 biofilms to deactivate toxic ions by absorption is a hallmark for the dual function of extracellular polymers: such extracellular polymers are typically responsible for tuning both the mechanical properties and the permeability of eukaryotic tissues. A very similar function is described here for a prokaryotic "tissue" where the biofilm matrix selectively traps toxic ions and - on top of that - even utilizes them to improve the mechanical performance of the biofilm layer. Thus, biofilm formation can be regarded as a well-tailored strategy that microorganisms use to survive in potentially harmful environments. ${ }^{54}$

\section{Acknowledgements}

We thank Masaaki Morikawa for providing the Bacillus subtilis B1 strain. This project was supported by the Deutsche Forschungsgemeinschaft through project B11 "Mechanics of bacterial biofilms" in the framework of SFB 863. Additional support from the Center for Nanoscience Munich (CeNS) and the International Graduate School of Science and Engineering (IGGSE) is gratefully acknowledged. The authors thank Konstantinia Bidmon for assistance with the bacterial cultures and Dionis Minev and Andreas Mader for conducting pilot experiments for this study. Marina Caldara's helpful comments on the manuscript are appreciated.

\section{References}

1 S. C. Booth, I. F. S. George, D. Zannoni, M. Cappelletti, G. E. Duggan, H. Ceri and R. J. Turner, Effect of aluminium and copper on biofilm development of Pseudomonas pseudoalcaligenes KF707 and P. fluorescens as a function of different media compositions, Metallomics, 2013, 5, 723-735.

2 A. Bridier, R. Briandet, V. Thomas and F. DuboisBrissonnet, Resistance of bacterial biofilms to disinfectants: a review, Biofouling, 2011, 27, 1017-1032.

3 J. J. Harrison, R. J. Turner and H. Ceri, High-throughput metal susceptibility testing of microbial biofilms, $B M C$ Microbiol., 2005, 5, 53.

4 J. Costerton, P. Stewart and E. Greenberg, Bacterial biofilms: A common cause of persistent infections, Science, 1999, 284, 1318-1322.

5 R. D. Monds and G. A. O'Toole, The developmental model of microbial biofilms: ten years of a paradigm up for review, Trends Microbiol., 2009, 17, 73-87.
6 G. O’Toole, H. B. Kaplan and R. Kolter, Biofilm formation as microbial development, Annu. Rev. Microbiol., 2000, 54, 49-79.

7 H. Vlamakis, Y. Chai, P. Beauregard, R. Losick and R. Kolter, Sticking together: building a biofilm the Bacillus subtilis way, Nat. Rev. Microbiol., 2013, 11, 157-168.

$8 \mathrm{~T}$. Bjarnsholt, The role of bacterial biofilms in chronic infections, APMIS, 2013, 121, 1-58.

9 S. R. Shah, A. M. Tatara, R. N. D’Souza, A. G. Mikos and F. K. Kasper, Evolving strategies for preventing biofilm on implantable materials, Mater. Today, 2013, 16, 177-182.

10 R. M. Donlan, Biofilms: Microbial Life on Surfaces, Emerging Infect. Dis., 2002, 8, 881-890.

11 B. Carpentier and O. Cerf, Biofilms and their consequences, with particular reference to hygiene in the food industry, J. Appl. Bacteriol., 1993, 75, 499-511.

12 S. Aggarwal and R. M. Hozalski, Effect of Strain Rate on the Mechanical Properties of Staphylococcus epidermidis Biofilms, Langmuir, 2012, 28, 2812-2816.

13 M. Böl, A. E. Ehret, A. Bolea Albero, J. Hellriegel and R. Krull, Recent advances in mechanical characterisation of biofilm and their significance for material modelling, Crit. Rev. Biotechnol., 2013, 33, 145-171.

14 A. W. Cense, E. A. G. Peeters, B. Gottenbos, F. P. T. Baaijem, A. M. Nuijs and M. E. H. van Dongen, Mechanical properties and failure of Streptococcus mutans biofilms, studied using a microindentation device, J. Microbiol. Methods, 2006, 67, 463-472.

15 D. N. Hohne, J. G. Younger and M. J. Solomon, Flexible Microfluidic Device for Mechanical Property Characterization of Soft Viscoelastic Solids Such as Bacterial Biofilms, Langmuir, 2009, 25, 7743-7751.

16 P. C. Y. Lau, J. R. Dutcher, T. J. Beveridge and J. S. Lam, Absolute Quantitation of Bacterial Biofilm Adhesion and Viscoelasticity by Microbead Force Spectroscopy, Biophys. J., 2009, 96, 2935-2948.

17 A. Rochex, A. Massé, R. Escudié, J.-J. Godon and N. Bernet, Influence of abrasion on biofilm detachment: evidence for stratification of the biofilm, J. Ind. Microbiol., 2009, 36, 467-470.

18 S. S. Rogers, C. van der Walle and T. A. Waigh, Microrheology of Bacterial Biofilms In Vitro: Staphylococcus aureus and Pseudomonas aeruginosa, Langmuir, 2008, 24, 13549-13555.

19 L. C. Simões, M. Lemos, P. Araújo, A. M. Pereira and M. Simões, The effects of glutaraldehyde on the control of single and dual biofilms of Bacillus cereus and Pseudomonas fluorescens, Biofouling, 2011, 27, 337-346.

20 Y. Zhou, A. Torres, L. Chen, Y. Kong, J. D. Cirillo and H. Liang, Fluid-shear method to evaluate bacterial adhesion to glass surfaces, J. Appl. Phys., 2012, 112, 014703.

$21 \mathrm{X}$. Chen and P. S. Stewart, Biofilm removal caused by chemical treatments, Water Res., 2000, 34, 4229-4233.

22 L. Pavlovsky, J. G. Younger and M. J. Solomon, In situ rheology of Staphylococcus epidermidis bacterial biofilms, Soft Matter, 2013, 9, 122-131.

23 M. J. Chen, Z. Zhang and T. R. Bott, Direct measurement of the adhesive strength of biofilms in pipes by micromanipulation, Biotechnol. Tech., 1998, 12, 875-880. 
24 X. Chen and P. Stewart, Role of electrostatic interactions in cohesion of bacterial biofilms, Appl. Microbiol. Biotechnol., 2002, 59, 718-720.

25 M. Wloka, H. Rehage, H.-C. Flemming and J. Wingender, Structure and rheological behaviour of the extracellular polymeric substance network of mucoid Pseudomonas aeruginosa biofilms, Biofilms, 2005, 2, 275-283.

26 W. L. Jones, M. P. Sutton, L. McKittrick and P. S. Stewart, Chemical and antimicrobial treatments change the viscoelastic properties of bacterial biofilms, Biofouling, 2011, 27, 207-215.

27 O. Lieleg, M. Caldara, R. Baumgaertel and K. Ribbeck, Mechanical robustness of Pseudomonas aeruginosa biofilms, Soft Matter, 2011, 7, 3307-3314.

28 P. Stoodley, L. Hall-Stoodley and H. Lappin-Scott, Microbial Growth In Biofilms, Pt B., Academic Press Inc, 525 B Street, Suite 1900, San Diego, CA 92101-4495 USA, 2001, vol. 337, pp. 306-318.

29 M. J. Chen, Z. Zhang and T. R. Bott, Effects of operating conditions on the adhesive strength of Pseudomonas fluorescens biofilms in tubes, Colloids Surf., B, 2005, 43, 61-71.

30 T. R. Garrett, M. Bhakoo and Z. Zhang, Characterisation of bacterial adhesion and removal in a flow chamber by micromanipulation measurements, Biotechnol. Lett., 2008, 30, 427-433.

31 P. Ramasamy and X. Zhang, Effects of shear stress on the secretion of extracellular polymeric substances in biofilms, Water Sci. Technol., 2005, 52, 217-223.

32 C. Rupp, C. Fux and P. Stoodley, Viscoelasticity of Staphylococcus aureus biofilms in response to fluid shear allows resistance to detachment and facilitates rolling migration, Appl. Environ. Microbiol., 2005, 71, 2175-2178.

33 K. M. Thormann, R. M. Saville, S. Shukla and A. M. Spormann, Induction of Rapid Detachment in Shewanella oneidensis MR-1 Biofilms, J. Bacteriol., 2005, 187, 1014-1021.

34 P. Stoodley, A. Jacobsen, B. Dunsmore, B. Purevdorj, S. Wilson, H. Lappin-Scott and J. Costerton, The influence of fluid shear and $\mathrm{AlCl}_{3}$ on the material properties of Pseudomonas aeruginosa PAO1 and Desulfovibrio sp. EX265 biofilms, Water Sci. Technol., 2001, 43, 113-120.

35 M. Simões, S. Cleto, M. O. Pereira and M. J. Vieira, Influence of biofilm composition on the resistance to detachment, Water Sci. Technol., 2007, 55, 473-480.

36 M. Simões, L. C. Simões and M. J. Vieira, Species association increases biofilm resistance to chemical and mechanical treatments, Water Res., 2009, 43, 229-237.

37 E. Banin, K. M. Brady and E. P. Greenberg, Chelator-Induced Dispersal and Killing of Pseudomonas aeruginosa Cells in a Biofilm, Appl. Environ. Microbiol., 2006, 72, 2064-2069.

38 A. Lomander, P. Schreuders, E. Russek-Cohen and L. Ali, Evaluation of chlorines' impact on biofilms on scratched stainless steel surfaces, Bioresour. Technol., 2004, 94, 275-283.

39 M. Simões, M. Pereira and M. Vieira, Effect of mechanical stress on biofilms challenged by different chemicals, Water Res., 2005, 39, 5142-5152.
40 H. A. Hong, R. Khaneja, N. M. K. Tam, A. Cazzato, S. Tan, M. Urdaci, A. Brisson, A. Gasbarrini, I. Barnes and S. M. Cutting, Bacillus subtilis isolated from the human gastrointestinal tract, Res. Microbiol., 2009, 160, 134-143.

41 M. Morikawa, Beneficial biofilm formation by industrial bacteria Bacillus subtilis and related species, J. Biosci. Bioeng., 2006, 101, 1-8.

42 J. C. Zweers, I. Barak, D. Becher, A. J. Driessen, M. Hecker, V. P. Kontinen, M. J. Saller, L. Vavrova and J. M. van Dijl, Towards the development of Bacillus subtilis as a cell factory for membrane proteins and protein complexes, Microb. Cell Fact., 2008, 7, 10.

43 M. Morikawa, M. Ito and T. Imanaka, Isolation of a New Surfactin Producer Bacillus-Pumilus a-1, and Cloning and Nucleotide-Sequence of the Regulator Gene, Psf-1, J. Ferment. Bioeng., 1992, 74, 255-261.

44 S. C. Andrews, A. K. Robinson and F. Rodrĩguez-Quiñones, Bacterial iron homeostasis, FEMS Microbiol. Rev., 2003, 27, 215-237.

45 P. Stoodley, R. Cargo, C. Rupp, S. Wilson and I. Klapper, Biofilm material properties as related to shear-induced deformation and detachment phenomena, J. Ind. Microbiol., 2002, 29, 361-367.

46 P. Sarin, V. L. Snoeyink, J. Bebee, K. K. Jim, M. A. Beckett, W. M. Kriven and J. A. Clement, Iron release from corroded iron pipes in drinking water distribution systems: effect of dissolved oxygen, Water Res., 2004, 38, 1259-1269.

47 H.-C. Flemming and J. Wingender, The biofilm matrix, Nat. Rev. Microbiol., 2010, 8, 623-633.

48 M. Morikawa, S. Kagihiro, M. Haruki, K. Takano, S. Branda, R. Kolter and S. Kanaya, Biofilm formation by a Bacillus subtilis strain that produces gamma-polyglutamate, Microbiology, 2006, 152, 2801-2807.

49 V. Körstgens, H. C. Flemming, J. Wingender and W. Borchard, Influence of calcium ions on the mechanical properties of a model biofilm of mucoid Pseudomonas aeruginosa, Water Sci. Technol., 2001, 43, 49-57.

50 E. R. Brindle, D. A. Miller and P. S. Stewart, Hydrodynamic Deformation and Removal of Staphylococcus epidermidis Biofilms Treated With Urea, Chlorhexidine, Iron Chloride, or DispersinB, Biotechnol. Bioeng., 2011, 108, 2968-2977.

51 I. W. Dawes, D. Kay and J. Mandelstam, Sporulation in Bacillus subtilis. Establishment of a time scale for the morphological events, J. Gen. Microbiol., 1969, 56, 171-179.

52 G. M. Teitzel and M. R. Parsek, Heavy metal resistance of biofilm and planktonic Pseudomonas aeruginosa, Appl. Environ. Microbiol., 2003, 69, 2313-2320.

53 S. Langley and T. J. Beveridge, Metal binding by Pseudomonas aeruginosa PAO1 is influenced by growth of the cells as a biofilm, Can. J. Microbiol., 1999, 45, 616-622.

54 J. J. Harrison, H. Ceri and R. J. Turner, Multimetal resistance and tolerance in microbial biofilms, Nat. Rev. Microbiol., 2007, 5, 928-938. 